\title{
Cloaca in Discordant Monoamniotic Twins: Prenatal Diagnosis and Consequence for Fetal Lung Development
}

\author{
Yvon Chitrit, MD ${ }^{1}$ Edith Vuillard, MD ${ }^{1}$ Sunavy Khung, MD, $\mathrm{PhD}^{2}$ Nadia Belarbi, MD ${ }^{3}$ \\ Fabien Guimiot, $\mathrm{PhD}^{2}$ Francoise Muller, MD, $\mathrm{PhD}^{4}$ Alaa El Ghoneimi, MD, $\mathrm{PhD}^{5}$ \\ Jean Francois Oury, MD, PhD ${ }^{1}$
}

${ }^{1}$ Department of Obstetrics and Gynecology, Robert Debré HospitalAP-HP, Paris, France

Address for correspondence Dr. Y. Chitrit, MD, Department of Obstetrics and Gynecology, Robert Debré Hospital, 48 Bd Sérurier,

2 Department of Developmental Biology, Robert Debré Hospital-AP75935 Paris cedex 19, France (e-mail: yvon.chitrit@rdb.aphp.fr).

HP, Paris, France

${ }^{3}$ Department of Pediatric Imaging, Robert Debré Hospital-AP-HP, Paris, France

${ }^{4}$ Laboratory of Biochemistry and Hormonology, Robert Debré Hospital-AP-HP, Paris, France

${ }^{5}$ Department of Pediatric Urology and Surgery, Robert Debré HospitalAP-HP, Paris, France

Am J Perinatol Rep 2014;4:33-36.

\begin{abstract}
Keywords

- prenatal diagnosis

- persistent cloaca

- monoamniotic twins

- fetal lung development

- discordant

Objective Describe a case of cloaca prenatally diagnosed in one of a set of monoamniotic twins.

Study Design Retrospective review of a case.

Results Cloaca is one of the most complex and severe degrees of anorectal malformations in girls. We present a discordant cloaca in monoamniotic twins. Fetal ultrasound showed a female fetus with a pelvic midline cystic mass, a phallus-like structure, a probable anorectal atresia with absence of anal dimple and a flat perineum, and renal anomalies. The diagnosis was confirmed by fetal magnetic resonance imaging postnatally.

Conclusions The rarity of the malformation in a monoamniotic pregnancy, the difficulties of prenatal diagnosis, the pathogenic assumptions, and the consequences of adequate amniotic fluid for fetal lung development are discussed.
\end{abstract}

Cloaca is one of the most complex and severe degrees of anorectal malformations in girls, in which the vagina, rectum, and urinary tract merge into a common channel leading to a single external orifice. We report a case of cloaca prenatally diagnosed in one of a set of monoamniotic twins. This observation underlines the difficulties of making a precise prenatal diagnosis and confirms the impact of an adequate amniotic fluid volume on fetal lung development.

\section{Case Report}

A 21-year-old mother, gravida 2, para 0 , presenting with a spontaneous monochorionic monoamniotic pregnancy was referred at 21 weeks because of a suspected urinary tract anomaly in twin A. The couple had no personal or family history of congenital anomalies . At successive ultrasound scans, the amnionicity and normality of the female twin B were confirmed. Twin A presented several following anomalies (-Fig. 1): a phallus-like structure measuring $15 \mathrm{~mm}$, a pelvic midline cystic mass, an absence of anal dimple, a flat perineum and renal anomalies combining left kidney agenesis, and a multicystic small right kidney. Amniotic fluid volume was normal on repeat scans (with a deepest vertical pocket at 21, 26, 32, and 34 weeks always less than $80 \mathrm{~mm}$ ). Fetal blood sampling performed at 22 weeks in twin A revealed a 46,XX karyotype. Fetal serum $\beta_{2}$-microglobulin received

September 27, 2013

accepted after revision

December 30, 2013

published online

March 3, 2014
Dol http://dx.doi.org/

10.1055/s-0034-1370351. ISSN 2157-6998.
Copyright (c) 2014 by Thieme Medical Publishers, Inc., 333 Seventh Avenue, New York, NY 10001, USA. Tel: +1(212) 584-4662.
License terms

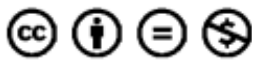




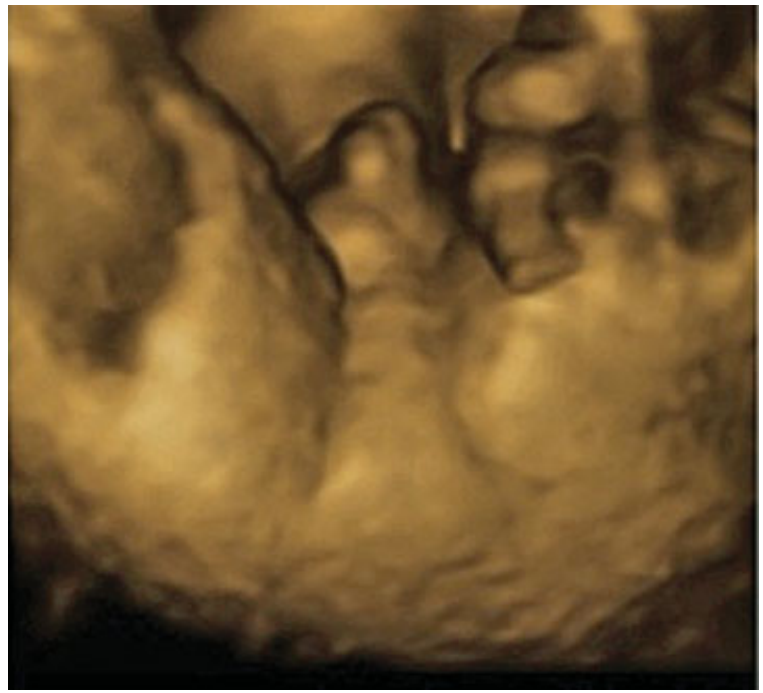

Fig. 1 Three-dimensional sonogram at 29 weeks of gestation shows a phallus-like structure, absence of anal dimple, and a flat perineum.

assayed for renal function evaluation was normal $(4.4 \mathrm{mg} / \mathrm{L}$ for a normal value $<5 \mathrm{mg} / \mathrm{L}$ ). Amniocentesis confirmed a 46 , XX fetal karyotype. Amniotic fluid 17-hydroxyprogesterone and digestive enzyme activities (gamma-glutamyl transpeptidase, aminopeptidase $\mathrm{M}$, and intestinal alkaline phosphatase) were normal, ${ }^{1}$ thus excluding congenital adrenal hyperplasia and digestive tract anomalies, respectively. Magnetic resonance imaging (MRI) of twin A showed an enlarged bladder associated with a fluid structure between the bladder and the spine but no T1-weighted high signal corresponding

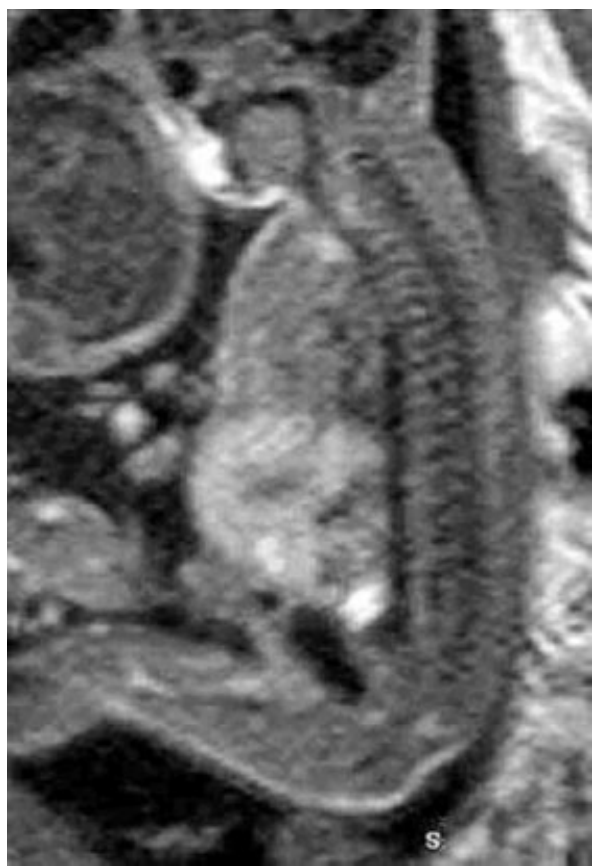

Fig. 2 Twin A magnetic resonance imaging: Absence of the T1-weighted high signal corresponding to the rectum.

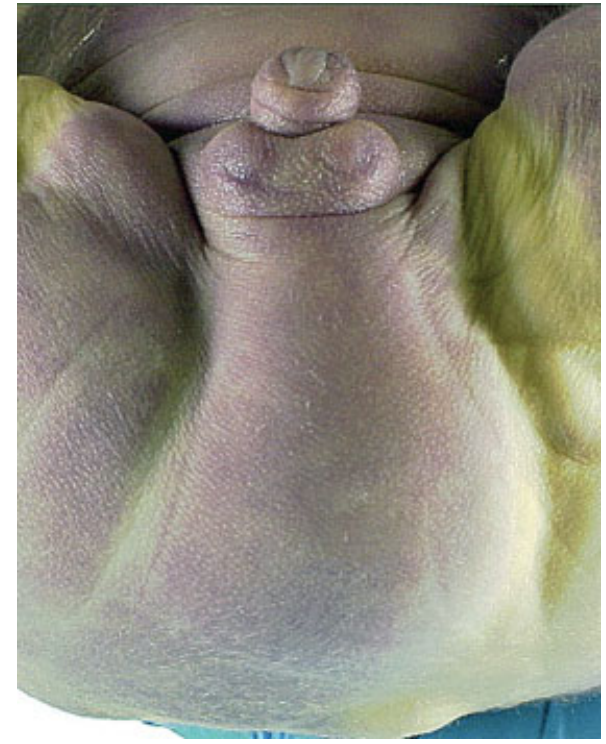

Fig. 3 Gross examination of the affected fetus: phallus-like structure, absence of anal dimple, and flat perineum.

to the rectum (-Fig. 2). Renal anomalies were confirmed. These findings were consistent with the diagnosis of cloaca. After prenatal counselling, selective termination of pregnancy was considered but not chosen by the parents.

A cesarean section was performed at 36 weeks. The female twin B $(2,010 \mathrm{~g})$ had no malformation. Twin A (2,310 g) presented a phallus-like structure measuring $11.5 \mathrm{~mm}$ with a single orifice at its extremity, a flat perineum with an imperforate anus, and no vaginal opening ( - Fig. 3 ). Left preaxial hexadactyly was also noted. Retrograde opacification achieved

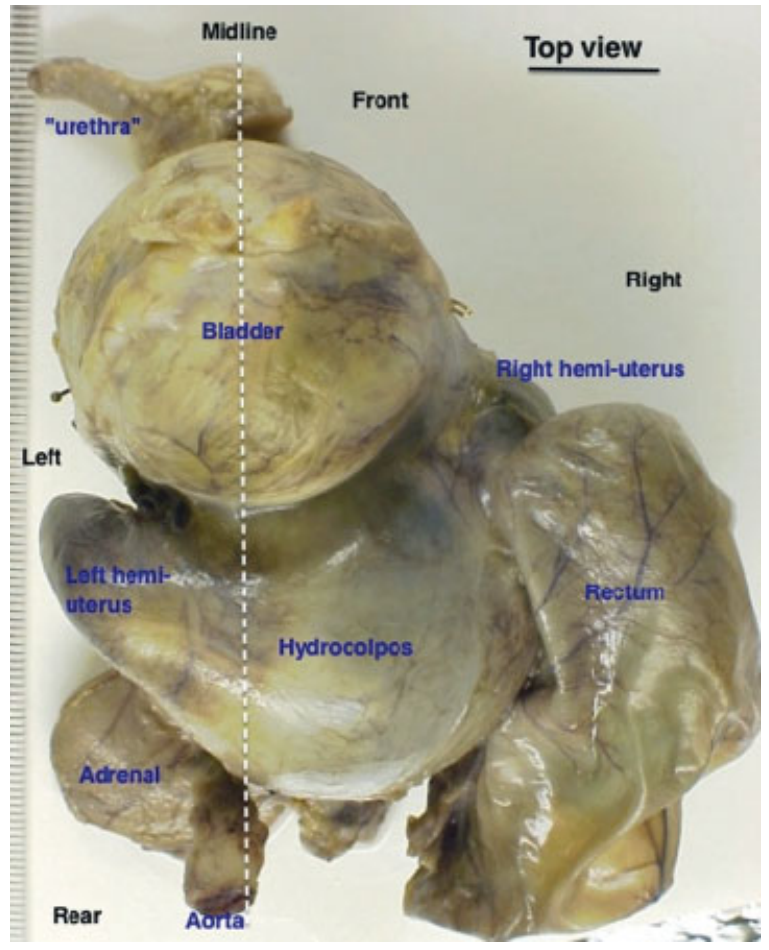

Fig. 4 Macroscopic examination of the affected fetus: the vagina, rectum, and urinary tract converge into a common channel leading to a single external orifice. 
through the single orifice demonstrated a communication between the "urethra" and a pelvic cavity. The neonate died at day 1 due to renal failure. Postmortem examination ( - Fig. 4) revealed that the urinary tract, the genital canal, and the rectum converged into a common channel, therefore confirming the diagnosis of cloaca. The bladder was distended by meconium. The consequent kidney abnormalities were confirmed. A sacral vertebral defect was discovered. These anomalies were consistent with caudal dysgenesis association. Interestingly, the lung volumes were normal.

\section{Comment}

Persistent cloaca ${ }^{2,3}$ (1 per 50,000 births), ${ }^{4,5}$ in which the genital canal, rectum, and urinary tract converge into a common channel leading to a single external orifice ${ }^{2,6}$ is one of the most complex and severe degrees of anorectal malformation. Hydrocolpos is present in $50 \%$ of these patients. Furthermore, cloaca is often associated with other anomalies, especially spinal and renal defects.

Anomalies of cloacal development involve the primitive hindgut and the caudal mesenchyme. Cloacal septation and rotation of the cloacal membrane, which swings down from a vertical to a horizontal position, involve two major processes $^{7}$ : active growth of the urorectal mesenchyme and fusion of the epithelial walls of the cloaca. These steps lead to a significant caudal lengthening of the so-called urorectal septum and produce an apparent septation of the cloaca. Later, regression of both the tailgut and the dorsal cloaca, and ventral protrusion of the urogenital sinus are the other major changes in the cloaca configuration. A defective cloacal anlage could result in a missing or misplaced anal orifice and abnormal communication between the rectum and the urogenital tract. ${ }^{8}$ It has been suggested ${ }^{9}$ that abnormal notochord development may induce neural tube defects and/or anorectal malformations.

To our knowledge, our case is the third occurrence of cloaca prenatally diagnosed in one of monozygotic twins. ${ }^{10,11}$ In addition, we observed that the affected twin lacked functional renal tissue but had a normal lung volume, therefore demonstrating that a sufficient amount of amniotic fluid produced by a monoamniotic twin environment could prevent pulmonary hypoplasia. This case report, such as the previous ones, provides an opportunity to highlight the importance of adequate amniotic fluid, ${ }^{12}$ among several other factors, for normal lung development. The normal fetal serum $\beta_{2}$-microglobulin we observed in the affected fetus is explained by the placental vascular anastomoses. Similarly, in this case, amniotic fluid digestive enzymes were paradoxically normal despite the presence of an urodigestive fistula in one of the two fetuses. ${ }^{13}$ This might be due to dilution and swallowing by the normal co-twin, which could have clarified the amniotic fluid. An another hypothesis could be the narrowness of the long and thin "urethra."

Anorectal malformations are difficult to diagnose prenatally. In a large series of 50 cases, ${ }^{4}$ cloaca was prenatally diagnosed at ultrasound scan in only three cases. The most common finding is a midline pelvic cystic mass located behind the bladder. ${ }^{3,4,14}$ If the cystic mass is septated, coni- cally shaped and/or contains sediments or calcifications, the likelihood of a cloaca increases. Other sonographic abnormalities $^{3}$ are highly suggestive of cloaca: bowel dilatations or calcifications, genitourinary (renal cyst, absent kidney, hydronephrosis, and ambiguous genitalia), and lumbosacral anomalies. In addition to these findings, the failure to see the anorectal tract by fetal sonography or MRI is very suggestive of cloaca. However, confounding diagnoses for a female fetus must be ruled out based on ultrasound scan, and/ or MRI: ovarian cyst (usually off midline), bladder obstruction (no debris within the cystic mass), intestinal duplication (thick echogenic wall and peristalsis), megacystis microcolon intestinal hypoperistaltsis syndrome (megacystis and absence of meconium in the rectum), isolated hydrocolpos, or urogenital sinus (meconium in the rectum). Amniotic fluid digestive enzymes and cyst fluid biochemistry ${ }^{13}$ could be of help to differentiate these diagnoses.

Congenital anomalies, concordant, or discordant, occur in approximately $10 \%$ of monozygotic twins. Several postfertilization $^{15,16}$ mechanisms have been proposed to explain discordant malformations in monozygotic twins such as chromosomal mosaicism, skewed X-inactivation, imprinting, postzygotic dominant or recessive gene mutation, and other epigenetic processes. For discordant cloacal dysgenesis, it has been hypothesized that there is an unequal allocation of stem cells to each twin, related to the twinning process itself, leading to an unequal potential of development and/or abnormal determination of laterality gradients. ${ }^{15}$

In conclusion, this case of cloaca in discordant monoamniotic twins underlines the difficulties of making a precise prenatal diagnosis and of analyzing biochemical results because of the vascular and amniotic communications between the twins. This case confirms the impact of an adequate amniotic fluid volume on fetal lung development.

\section{Conflict of Interest}

The authors declare no conflict of interest.

\section{References}

1 Muller F, Dommergues M, Ville Y, et al. Amniotic fluid digestive enzymes: diagnostic value in fetal gastrointestinal obstructions. Prenat Diagn 1994;14(10):973-979

2 Levitt MA, Peña A. Anorectal malformations. Orphanet J Rare Dis 2007;2:33-44

3 Winkler NS, Kennedy AM, Woodward PJ. Cloacal malformation: embryology, anatomy, and prenatal imaging features. J Ultrasound Med 2012;31(11):1843-1855

4 Livingston JC, Elicevik M, Breech L, Crombleholme TM, Peña A, Levitt MA. Persistent cloaca: a 10-year review of prenatal diagnosis. J Ultrasound Med 2012;31(3):403-407

5 Warne SA, Hiorns MP, Curry J, Mushtaq I. Understanding cloacal anomalies. Arch Dis Child 2011;96(11):1072-1076

6 Petrikovsky BM, Walzak MP Jr, D’Addario PF. Fetal cloacal anomalies: prenatal sonographic findings and differential diagnosis. Obstet Gynecol 1988;72(3 Pt 2):464-469

7 Qi BQ, Williams A, Beasley S, Frizelle F. Clarification of the process of separation of the cloaca into rectum and urogenital sinus in the rat embryo. J Pediatr Surg 2000;35(12):1810-1816 
36 Cloaca in Discordant Twins Chitrit et al.

8 Kluth D. Embryology of anorectal malformations. Semin Pediatr Surg 2010;19(3):201-208

9 Qi BQ Beasley SW, Frizelle FA. Evidence that the notochord may be pivotal in the development of sacral and anorectal malformations. J Pediatr Surg 2003;38(9):1310-1316

10 Meyer SR, Smith KM, Ravish IR, Saltzman DA, Shukla AR. Complex cloacal anomaly in discordant monozygotic twins. Urology 2009; 73(3):532-534

11 Achiron R, Frydman M, Lipitz S, Zalel Y. Urorectal septum malformation sequence: prenatal sonographic diagnosis in two sets of discordant twins. Ultrasound Obstet Gynecol 2000;16(6): 571-574

12 Adzick NS, Harrison MR, Glick PL, Villa RL, Finkbeiner W. Experimental pulmonary hypoplasia and oligohydramnios: relative con- tributions of lung fluid and fetal breathing movements. J Pediatr Surg 1984;19(6):658-665

13 Lecarpentier E, Dreux S, Blanc T, et al. Biochemical analysis of cystic fluid in the diagnosis of fetal intra-abdominal masses. Prenat Diagn 2012;32(7):627-631

14 Bischoff A, Levitt MA, Lim FY, Guimarães C, Peña A. Prenatal diagnosis of cloacal malformations. Pediatr Surg Int 2010; 26(11):1071-1075

15 Silva S, Martins Y, Matias A, Blickstein I. Why are monozygotic twins different? J Perinat Med 2011;39(2):195-202

16 Machin G. Non-identical monozygotic twins, intermediate twin types, zygosity testing, and the non-random nature of monozygotic twinning: a review. Am J Med Genet C Semin Med Genet 2009;151C(2):110-127 